\title{
An electronic atlas of human malformation syndromes in diverse populations
}

\author{
Maximilian Muenke, MD ${ }^{1}$, Adebowale Adeyemo, $\mathrm{MD}^{2}$ and Paul Kruszka, MD, MPH${ }^{1}$
}

\section{INTRODUCTION}

Birth defects remain a leading cause of infant mortality and childhood morbidity worldwide. As laboratory sequencing technologies become more available, recognition of malformation syndromes will become increasingly important. Most clinicians have been trained with clinical genetic resources that used patients of northern European descent as the standard of reference. The most well-known textbook of dysmorphology, Smith's Recognizable Patterns of Human Malformation, shows predominantly Caucasian patients. ${ }^{1}$ Additionally, medical journals containing syndromes often show only patients of European ancestry. This poses a problem when confronted with patients from other parts of the world because of the wide range of normal physical features across the globe. As an example, many cases of Williams syndrome have been reported in the medical literature, but few reports involved African Americans or sub-Saharan Africans. Not surprisingly, the diagnostic criteria for Williams syndrome does not take into account differences in ethnicities. ${ }^{2}$ Thus, the diagnosis can be difficult in non-European patients, as exemplified by a report from subSaharan Africa where experienced geneticists struggled to diagnose Williams syndrome. ${ }^{3}$ Facing this obstacle, Shotelersuk published a book of syndromes of Thai individuals to provide ethnic-specific cases for physicians practicing in Thailand. ${ }^{4}$ This book and a recent commentary $y^{4,5}$ revealed the difficulty in diagnosing single-gene disorders in different ethnicities given the variation of phenotypes and appearances. A systematic collection of dysmorphologic disorders across patients of different ethnicities would be highly valuable for clinical diagnosis, providing genetic counseling, estimating population prevalence, and conducting research.

We have had our own experiences in clinical genetics with challenges in identifying specific malformation syndromes in patients from different ethnicities. M.M. trained in pediatrics and genetics in Germany, where using textbooks with exclusive examples of photos of patients of northern European descent worked well, but later, while training at the Children's Hospital of Philadelphia, he realized that he had to relearn morphologic features as they applied to diverse populations. A.A. trained in clinical genetics in Nigeria, using medical literature that did not adequately represent the syndromic features seen in subSaharan Africa, which inspired his first publication in morphology. ${ }^{6}$ P.K. has traveled and consulted in genetics throughout the world and has come to realize the importance of diagnostic tools for diverse populations that could be accessed on mobile devices.

In this commentary, we introduce a freely available electronic photo atlas of individuals from diverse populations with human malformation syndromes. Photos for this website are previously unpublished and obtained from colleagues throughout the world. The core of this resource is a catalog of genetic and dysmorphologic syndromes with a molecular diagnosis, accompanied by photographs of affected patients of various ethnicities. This freely available clinical tool is located on the website for the National Human Genome Research Institute (NHGRI) (http://research.nhgri.nih.gov/ atlas). The atlas is searchable by phenotype, syndrome, ethnicity, and genetic/molecular diagnosis. To improve ease of access, the site will be optimized for mobile devices (such as smartphones and tablets), and an accompanying app will be developed.

This resource will become increasingly important as congenital malformations comprise a larger fraction of childhood morbidity and mortality, with congenital abnormalities already ranking as the fifth leading cause of death in neonates (age 1-27 days) and accounting for 270,000 deaths worldwide in 2010 (4\% of all deaths). ${ }^{7}$ With the rapid expansion of genetic and genomic medicine since completion of the Human Genome Project in 2003, Western countries have enjoyed these advances; however, in third-world countries where $80 \%$ of people live and $90 \%$ of births occur, there has been little impact. ${ }^{8}$ Genetic services have been traditionally available only at tertiary-care and university settings. In our expanding world, it is not possible for every individual with a genetic condition or a birth defect to have access to a clinical geneticist and a genetic counselor. Our tool will be well suited for use by both clinical geneticist and nongeneticist clinicians such as primary-care physicians, especially in developing countries.

Making a proper and early syndromic diagnosis is paramount because late diagnosis can result in a delay in intervention and

${ }^{1}$ Medical Genetics Branch, National Human Genome Research Institute, National Institutes of Health, Bethesda, Maryland, USA; ${ }^{2}$ Center for Research on Genomics and Global Health, National Human Genome Research Institute, National Institutes of Health, Bethesda, Maryland, USA. Correspondence: Maximilian Muenke (mamuenke@mail.nih.gov) 
treatment for accompanying malformations such as congenital heart defects or endocrine disorders. In 2010, the World Health Organization began to focus on congenital malformations and announced support for public health policy directed at preventing congenital malformations through various measures. ${ }^{9} \mathrm{We}$ anticipate that our electronic atlas will assist clinicians in associating congenital malformations with syndromes, allowing for earlier diagnosis and addressing the potential associated medical issues.

\section{DESIGN OF THE ELECTRONIC ATLAS}

The purpose of this website is to provide a tool that is easy to use and helpful for the clinician to make a diagnosis in syndromic disorders across diverse populations. Photographs of the face and other relevant body areas are the main focus. Accompanying information will include general phenotype description and genetic variants associated with each syndrome. The website includes photographs of individuals from geographically diverse locations, including locations in Asia, the Indian subcontinent, the Middle East, South America, and sub-Saharan Africa. The idea is that the website will be more of a photo atlas than a textbook, and it is designed to provide the kind of information obtainable from standard texts such as Smith's Recognizable Patterns of Malformation. ${ }^{1}$ The website is open-access and, as noted, it is accessible through the National Human Genome Research Institute website (http://www. research.nhgri.nih.gov/atlas).

\section{ADVISORY BOARD}

Oversight of the website and its contents is provided by an international board of experts in clinical genetics and dysmorphology. The advisory board consists of clinicians from the United States, Brazil, China, India, Japan, the Middle East, sub-Saharan Africa, and Thailand.

\section{ETHICAL CONSIDERATIONS}

No identifying personal information will appear on the website. There is a remote chance that one's private gene mutation could function as an identifying piece of information. Several ethical considerations must be addressed when launching a project of this magnitude that showcases dysmorphic features in diverse populations. For example, we will not obscure facial features, because facial features are crucial to the diagnosis. This means that a specific patient may be identifiable. However, this practice is no different from that for other textbooks of clinical genetics or malformation syndromes. In an accompanying article in this issue, Koretzky et al. ${ }^{10}$ explore these complex and important ethical topics.

\section{DISCUSSION}

We foresee that this tool for evaluating dysmorphic patients from a diverse population will be used extensively by physicians, genetic counselors, and trainees throughout the world. Web resources are slowly becoming more popular than paper textbooks among medical providers because they can be used at the point of care using only a cell phone. There are overlapping websites, including the Geneva Foundation for Medical Education and Research's database of developmental and genetic diseases (http://www.gfmer.ch/genetic_diseases_v2/ index.php) and the NHGRI's Elements of Morphology: Human Malformation Terminology (http://elementsofmorphology. nih.gov). Other websites featuring genetic and dysmorphology syndromes differ from ours in that their images have been previously published in journals or come from other databases. Our atlas focuses on representing the diversity of populations around the world in contrast to the usual representations seen in journals and textbooks.

The international clinical genetics community is small, and extensive use of the atlas will probably be universal. The challenge will be informing other physician communities, such as pediatricians, family physicians, internists, cardiologists, neurologists, and craniofacial surgeons. The open access and the location on the NHGRI website will be attractive to those in other specialties. Monitoring website parameters, such as visit numbers and syndrome type, will help maintain and promote this needed resource.

The tool, while not replacing superior clinical skills, is intended to broaden the phenotype of syndromes to diverse populations and assist trained clinicians to make diagnoses in these populations. Variance in expression is a common finding in malformation syndromes. This website will attempt to show multiple individuals from various populations with a specific syndrome, but it will not show every syndrome variation. Additionally, most major malformations are nonspecific to a particular syndrome; therefore, this atlas will attempt to show the patterns of malformations that are consistent with a syndrome.

\section{FUTURE DEVELOPMENTS}

This electronic resource will lead to the practical application of syndrome recognition, enabling appropriate intervention, including genetic counseling and screening of other organ systems. Additional developments can be built on this resource, including:

- A mobile app that has all the functionality of the Web portal can make this atlas even more user-friendly, given the ubiquity of smartphones all over the world.

- The images can serve as a resource for developing facerecognition software that could potentially improve diagnosis of specific syndromes. Having such software means that even when a specialist is not available, clinicians can take a photograph using their smartphones and the software can provide a differential diagnosis.

- The network of clinicians contributing to and using this resource can more easily collaborate on research questions and clinical trials for specific syndromes, which, individually, are usually rare. 


\section{ACKNOWLEDGMENTS}

This work was supported by the Division of Intramural Research of the National Human Genome Research Institute at the National Institutes of Health..

\section{DISCLOSURE}

The authors declare no conflict of interest.

\section{REFERENCES}

1. Jones, LJ, Jones MC, Del Campo M. Smith's Recognizable Patterns of Human Malformation. 7th edn. Elsevier Saunders: Philadelphia, PA, 2013.

2. Committee on Genetics. American Academy of Pediatrics: health care supervision for children with Williams syndrome. Pediatrics 2001;107: 1192-1204; erratum, 2002;109:329.

3. Tekendo-Ngongang C, Dahoun S, Nguefack S, Gimelli S, Sloan-Béna F, Wonkam A. Challenges in clinical diagnosis of Williams-Beuren syndrome in subSaharan Africans: case reports from Cameroon. Mol Syndromo/ 2014:5:287-292.

4. Shotelersuk, V. Clinical Genetics in Children. Text and Journal Publication. Bangkok, Thailand, 2003.
5. Shotelersuk V, Limwongse C, Mahasirimongkol S. Genetics and genomics in Thailand: challenges and opportunities. Mol Genet Genomic Med 2014;2: 210-216.

6. Adeyemo $\mathrm{AA}$, Omotade $\mathrm{OO}$. Variation in fontanelle size with gestational age. Early Hum Dev 1999;54:207-214

7. Liu L, Johnson HL, Cousens S, et al.; Child Health Epidemiology Reference Group of WHO and UNICEF. Global, regional, and national causes of child mortality: an updated systematic analysis for 2010 with time trends since 2000. Lancet 2012;379:2151-2161.

8. Report of the joint World Health Organization (WHO)/World Alliance of Organizations for the Prevention of Birth Defects (WAOPBD) meeting (WHO/ HGN/GL/WAOPBD/99.1). Services for the Prevention and Management of Genetic Disorders and Birth Defects in Developing Countries. World Health Organization: Geneva, Switzerland, 1999.

9. Resolution WHA63.17. Birth defects. In: Sixty-third World Health Assembly, Geneva, 17-21 May 2010. vol. 1. Resolutions and decisions. World Health Organization: Geneva, Switzerland, 2010 (WHA63/2010/REC/1). http://apps. who.int/gb/ebwha/pdf files/WHA63-REC1NHHA63_REC1-en.pdf. Accessed 17 July 2015.

10. Koretzky M, Bonham VL, Berkman BE, et al. Toward a more representative morphology: clinical and ethical considerations for including diverse populations in diagnostic genetic atlases. Genet Med 2016;18: in press. 